\title{
ON THE TOPOLOGY OF REAL BOTT MANIFOLDS
}

\author{
RAISA DSOUZA
}

\begin{abstract}
The main aim of this article is to give a necessary and sufficient condition for a real Bott manifold to admit a spin structure and further give a combinatorial characterization for the spin structure in terms of the associated acyclic digraph.
\end{abstract}

Keywords: Real Bott manifolds, orientability, spin structure, oriented null-cobordism.

\section{INTRODUCTION}

A Bott tower is an iterated sequence of fibre bundles with fibre at each stage being $\mathbb{C P}^{1}$. The manifold at each stage of the sequence is called a Bott manifold. In particular these manifolds are smooth projective complex toric varieties. They were constructed in [9] by Grossberg and Karshon who show that a Bott-Samelson variety can be deformed to a Bott manifold. Apart from [9], the topology and geometry of these objects have been studied by Civan and Ray in 4] and [5]. In particular, in 44, Civan looks at these manifolds as a special class of smooth toric varieties and studies their construction in terms of Bott numbers.

Indeed, from the viewpoint of toric topology, Bott manifolds can be seen to also have the structure of a quasitoric manifold [6] with the quotient polytope being the $n$-dimensional cube $I^{n}$ where $n$ is the complex dimension of the Bott manifold.

Recently there has been extensive work on the topology and geometry of Bott manifolds viewed as a quasitoric manifold (see [18] and [3]). These works are especially related to the problem of cohomological rigidity of Bott manifolds or more generally of quasitoric manifolds.

There has also been a parallel study on the topology of real Bott manifolds. These manifolds are constructed as iterated $\mathbb{R} \mathbb{P}^{1}=S^{1}$-bundles and can be viewed as a special example of a small cover defined by Davis and Januszkiewicz in [6] (see for example [12, [2] ). Moreover, the data of the characteristic function for this small cover is encoded by an upper triangular nilpotent matrix $C \in M_{n}\left(\mathbb{Z}_{2}\right)$. We call $C$ the Bott matrix and its entries $c_{i, j} 1 \leq i<j \leq n$, Bott numbers.

It is known [12] that a real Bott manifold is orientable if and only if

$$
\sum_{j=1}^{n} c_{i, j} \equiv 0 \bmod 2 \quad \text { for } \quad 1 \leq i \leq n .
$$

Indeed (1.1) is equivalent to the vanishing of the first Stiefel-Whitney class of the real Bott manifold in the cohomology ring with $\mathbb{Z}_{2}$-coefficients. Our motivation in this article is to give necessary and sufficient conditions for the vanishing of the higher Stiefel-Whitney classes in

${ }^{0} 2010$ Subject Classification: Primary: 55R99, Secondary: 57S25 
terms of algebraic identities in the $c_{i, j}$ 's. This is of interest since the vanishing of the StiefelWhitney classes have topological interpretations. In particular, in Theorem 3.2 we derive a closed formula for the second Stiefel-Whitney class in the $c_{i, j}$ 's and hence obtain a necessary and sufficient condition for a real Bott manifold to be spin.

In Section 3.1, we consider a more general real Bott manifold $M(B)$, where $B=\left(b_{i, j}\right) \in \mathcal{B}(n)$ [2, Section 1, page 2], and give a necessary and sufficient condition in Theorem $\underline{3.12}$ for $M(B)$ to admit a spin structure in terms of algebraic identities on the $b_{i, j}$ 's. We wish to mention here that the spin structure of real Bott manifolds has recently been studied by Gassior in [8], where again a necessary and sufficient condition has been given for $M(B)$ to be spin. We would like to point out that our characterization in Theorem $\underline{3.12}$ is more intrinsic and the main result [8, Theorem 1.2] follows as a corollary (see Corollary 3.13).

In 2], Choi, Masuda and Oum associate an acyclic directed graph to a real Bott manifold. Indeed this is a directed graph whose adjacency matrix is the Bott matrix. Apart from other results, in [2, Lemma 4.1], they give a criterion for orientability and symplectic structure on the Bott manifold in terms of the combinatorics of the associated digraph. Motivated by their work we obtain a digraph-characterization for the spin structure on these manifolds in Theorem 4.5,

In Theorem 5.1 we give a formula for the $(n-1)^{\text {th }}$ Stiefel-Whitney class. Further, in Theorem 5.5 we prove that real Bott manifolds are null-cobordant by showing that all their Stiefel-Whitney numbers vanish. Moreover, in Corollary 5.7 we prove that an oriented real Bott manifold is orientedly null-cobordant.

1.1. Notations and Conventions. In this section we recall the definition of a Bott tower and fix some notations (see [9]).

A Bott tower is a sequence of smooth complete complex toric varieties which are constructed iteratively as follows:

Let $Y_{1}=\mathbb{C P}^{1}$. Let $L_{2}$ be a holomorphic complex line bundle on $\mathbb{C P}^{1}$. We then let $Y_{2}=$ $\mathbb{P}\left(\mathbf{1} \oplus L_{2}\right)$ where $\mathbf{1}$ is the trivial line bundle on $\mathbb{C P}^{1}$. Then $Y_{2}$ is a $\mathbb{C P}^{1}$ bundle over $\mathbb{C P}^{1}$ which is a Hirzebruch surface. We can iterate this process for $2 \leq j \leq n$, where at each step, $L_{j}$ is a complex line bundle over $Y_{j-1}$, and the variety $Y_{j}=\mathbb{P}\left(\mathbf{1} \oplus L_{j}\right)$ is a $\mathbb{C P}^{1}$ bundle over $Y_{j-1}$. The sequence

$$
Y_{n} \rightarrow Y_{n-1} \rightarrow \cdots \rightarrow Y_{1} \rightarrow\{\mathrm{pt}\}
$$

is called an $n$-step Bott tower. Each $Y_{j}$ is called a Bott manifold.

Definition 1.1. In fact an $n$-dimensional Bott manifold is a smooth complete toric variety of dimension $n$ whose fan $\Delta$ can be described as follows: 
We take a collection of integers $\left\{a_{i, j}\right\}, 1 \leq i<j \leq n$. Let $e_{1}, \cdots, e_{n}$ be the standard basis vectors of $\mathbb{R}^{n}$. Let $v_{j}=e_{j}$ for $1 \leq j \leq n$,

$$
v_{n+j}=-e_{j}+\sum_{k=j+1}^{n} a_{j, k} e_{k}
$$

for $1 \leq j \leq n-1$ and $v_{2 n}=-e_{n}$. We define the fan $\Delta$ in $\mathbb{R}^{n}$ consisting of cones generated by the set of vectors in any sub collection of $\left\{v_{1}, v_{2}, \cdots, v_{n}, v_{n+1}, \cdots, v_{2 n}\right\}$ which does not contain both $v_{i}$ and $v_{n+i}$ for $1 \leq i \leq n$.

Definition 1.2. We can also view a Bott manifold as a quasi-toric manifold (see [6]) over the $n$-cube $I^{n}$ which is a simple convex polytope of dimension $n$. If we index the $2 n$ facets of $I^{n}$ by $F_{1}, F_{2}, \ldots, F_{n}, F_{n+1}, \ldots, F_{2 n}$, then the characteristic function, $\lambda$ is defined on the collection of facets, $\mathcal{F}$ to $\mathbb{Z}^{n}$ as follows: $\lambda\left(F_{j}\right)=e_{j}$ for $1 \leq j \leq n$,

$$
\lambda\left(F_{n+j}\right)=-e_{j}+\sum_{k=j+1}^{n} a_{j, k} \cdot e_{j+k}
$$

for $1 \leq j \leq n-1$ and $\lambda\left(F_{2 n}\right)=-e_{n}$.

1.1.1. Real Bott manifold. We shall call the real part of the $n$-dimensional complex Bott manifold as the real $n$-dimensional Bott manifold.

In particular, $\left(Y_{2}\right)_{\mathbb{R}}$ is an $\mathbb{R P}^{1}$ bundle over $\left(Y_{1}\right)_{\mathbb{R}}=\mathbb{R} \mathbb{P}^{1}$. Iteratively we construct $\left(Y_{j}\right)_{\mathbb{R}}$ as an $\mathbb{R P}^{1}$ bundle over $\left(Y_{j-1}\right)_{\mathbb{R}}$ for $2 \leq j \leq n$. The real $n$-dimensional Bott manifold $\left(Y_{n}\right)_{\mathbb{R}}$ is indeed the real toric variety associated to the fan $\Delta$ described above (see [11, Section 2.4] and [22]).

Definition 1.3. As in the complex case we can also view $\left(Y_{n}\right)_{\mathbb{R}}$ as a small cover over the simple convex polytope $I^{n}$, where the characteristic map $\lambda$ is defined on the collection of facets, $\mathcal{F}$ to $\mathbb{Z}_{2}^{n}$ as follows: $\lambda\left(F_{j}\right)=e_{j}$ for $1 \leq j \leq n$,

$$
\lambda\left(F_{n+j}\right)=e_{j}+\sum_{k=j+1}^{n} c_{j, k} \cdot e_{k}
$$

for $1 \leq j \leq n-1$ and $\lambda\left(F_{2 n}\right)=e_{n}$ where $c_{i, j}=a_{i, j} \bmod 2$ for $1 \leq i<j \leq n$. Thus $\left(Y_{n}\right)_{\mathbb{R}}$ is homeomorphic to the identification space $\mathbb{Z}_{2}^{n} \times I^{n} / \sim$ where $(t, p) \sim\left(t^{\prime}, p^{\prime}\right)$ if and only if $p=p^{\prime}$ and $t \cdot\left(t^{\prime}\right)^{-1} \in G_{F(p)}$. Here $F(p)=F_{1} \cap \cdots \cap F_{l}$ is the unique face of $I^{n}$ which contains $p$ in its relative interior and $G_{F(p)}$ is the rank-l subgroup of $\mathbb{Z}_{2}^{n}$ determined by the span of $\lambda\left(F_{1}\right), \ldots, \lambda\left(F_{l}\right)$.

The topological structure of an $n$-dimensional real Bott manifold is completely determined by the simple convex polytope $I^{n}$ and the data encoded by the matrix

$$
C=\left(c_{i, j}\right) \in M_{n}\left(\mathbb{Z}_{2}\right)
$$

where $c_{i, j}=0$ for $i \geq j$. Note that the $i$ th row of $C+I$ is $\lambda\left(F_{n+i}\right) \in \mathbb{Z}_{2}^{n}$ for $1 \leq i \leq n$. We call $C$ the Bott matrix. Thus $\left(Y_{n}\right)_{\mathbb{R}}$ is the real Bott manifold associated to $C$. 
The 2-dimensional real Bott manifold is the torus or the Klein bottle depending on whether $c_{1,2}=0$ or $c_{1,2}=1$. The 3 -dimensional real Bott manifold is an $\mathbb{R P}^{1}$ bundle over the torus or the Klein bottle whose topological structure depends on $c_{1,2}, c_{1,3}$ and $c_{2,3}$.

Note. In this article, since we are mainly interested in the study of real Bott manifolds, for notational simplicity we shall henceforth denote $\left(Y_{n}\right)_{\mathbb{R}}$ by $Y_{n}$. If we wish to specify the associated Bott matrix we shall denote $Y_{n}$ by $Y_{n}(C)$.

\section{Stiefel-Whitney Classes of $Y_{n}$}

In this section we briefly recall the description of the cohomology ring with $\mathbb{Z}_{2}$-coefficients of $Y_{n}$. A discription of $H^{*}\left(Y_{n} ; \mathbb{Z}_{2}\right)$ has been given earlier in [12, Section 2]. We also recall the formula for total Stiefel-Whitney class as given in [6, Section 6]. In Theorem [2.3 we give a recursive formula for the total Steifel-Whitney class as well as the $k$ th Steifel-Whitney class of $Y_{n}$ in terms of those of $Y_{n-1}$.

Proposition 2.1. Let $\mathcal{R}:=\mathbb{Z}_{2}\left[x_{1}, x_{2}, \cdots, x_{2 n}\right]$ and let $\mathcal{I}$ denote the ideal in $\mathcal{R}$ generated by the following set of elements

$$
\left\{x_{j} x_{n+j}, x_{1}+x_{n+1}, x_{j}+x_{n+j}+\sum_{i=1}^{j-1} c_{i, j} x_{n+i} \forall 2 \leq j \leq n\right\}
$$

As a graded $\mathbb{Z}_{2}$-algebra, $H^{*}\left(Y_{n} ; \mathbb{Z}_{2}\right)$ is isomorphic to $\mathcal{R} / \mathcal{I}$.

Let $w_{k}\left(Y_{n}\right)$ denote the $k^{\text {th }}$ Stiefel-Whitney class of $Y_{n}$ for $0 \leq k \leq n$ with the understanding that $w_{0}\left(Y_{n}\right)=1$. Then $w\left(Y_{n}\right)=1+w_{1}\left(Y_{n}\right)+\cdots+w_{n}\left(Y_{n}\right)$ is the total Stiefel-Whitney class of $Y_{n}$.

Proposition 2.2. (i) Under the isomorphism of $H^{*}\left(Y_{n} ; \mathbb{Z}_{2}\right)$ with $\mathcal{R} / \mathcal{I}$ we have the identification

$$
w\left(Y_{n}\right)=\prod_{i=1}^{2 n}\left(1+x_{i}\right)
$$

where $x_{i}$ for $1 \leq i \leq 2 n$ satisfy (2.1).

(ii) We further have the following recursive formula

$$
w\left(Y_{n}\right)=w\left(Y_{n-1}\right) \cdot\left(1+x_{n}\right)\left(1+x_{2 n}\right),
$$

where

$$
x_{n} \cdot x_{2 n}=0, x_{n}=x_{2 n}-\sum_{i=1}^{n-1} c_{i, n} x_{n+i} .
$$

Proof. The proof of (i) follows readily by applying [6, Corollary 6.8] for $Y_{n}$.

Now, we shall prove (ii).

Note that the defining Bott matrix for $Y_{n-1}$ is the $(n-1) \times(n-1)$ submatrix of $C$ obtained by deleting the $n$th row and the $n$th column of $C$. Moreover, let $\pi_{n}: Y_{n} \rightarrow Y_{n-1}$ denote 
the canonical projection of the $\mathbb{R P}^{1}$-bundle. Then via pullback along $\pi_{n}^{*}, H^{*}\left(Y_{n-1} ; \mathbb{Z}_{2}\right)$ can be identified with the subring $\mathcal{R}^{\prime} / \mathcal{I}^{\prime}$ of $\mathcal{R} / \mathcal{I}$ where $\mathcal{R}^{\prime}=\mathbb{Z}_{2}\left[x_{1}, x_{2} \ldots, x_{n-1}, x_{n+1}, \ldots, x_{2 n-1}\right]$ and $\mathcal{I}^{\prime}$ is the ideal generated by the relations

$$
\left\{x_{i} x_{n+i}, x_{i}-x_{n+i}+\sum_{j=1}^{i-1} c_{j, i} \cdot x_{n+j} \text { for } 1 \leq i \leq n-1\right\} .
$$

Since $Y_{n}$ is an $\mathbb{R P}^{1}$-bundle over $Y_{n-1}$, we further have the following presentation of $H^{*}\left(Y_{n} ; \mathbb{Z}_{2}\right)$ as an algebra over $H^{*}\left(Y_{n-1} ; \mathbb{Z}_{2}\right)$ :

$$
H^{*}\left(Y_{n} ; \mathbb{Z}_{2}\right) \simeq H^{*}\left(Y_{n-1} ; \mathbb{Z}_{2}\right)\left[x_{n}, x_{2 n}\right] / J
$$

where $J$ is the ideal generated by the relations

$$
x_{n} \cdot x_{2 n}, \quad x_{n}-x_{2 n}+\sum_{i=1}^{n-1} c_{i, n} x_{n+i} .
$$

Furthermore, via $\pi_{n}^{*}$ we can identify $w\left(Y_{n-1}\right)$ with the expression

$$
w\left(Y_{n-1}\right)=\prod_{i=1}^{n-1}\left(1+x_{i}\right) \cdot \prod_{i=n+1}^{2 n-1}\left(1+x_{i}\right)
$$

in $\mathcal{R}^{\prime}$ where $x_{i}$ for $1 \leq i \leq n-1$ and $n+1 \leq i \leq 2 n-1$ satisfy the relations (2.5). Now by (2.2) and (2.8), (ii) follows.

Theorem 2.3. $\quad$ (i) The following hold in the $\mathbb{Z}_{2}$-algebra $\mathcal{R} / \mathcal{I}$ :

$$
\begin{gathered}
w\left(Y_{n}\right)=w\left(Y_{n-1}\right)\left(1+\sum_{i=1}^{n-1} c_{i, n} x_{n+i}\right), \\
w_{k}\left(Y_{n}\right)=w_{k}\left(Y_{n-1}\right)+w_{k-1}\left(Y_{n-1}\right) \cdot\left(\sum_{i=1}^{n-1} c_{i, n} x_{n+i}\right)
\end{gathered}
$$

for $n \geq 2$ and $1 \leq k \leq n$.

(ii) For every $1 \leq k \leq n, w_{k}\left(Y_{n}\right)$ is a $\mathbb{Z}_{2}$-linear combination of square free monomials of degree $k$ in the variables $x_{n+1}, \ldots, x_{2 n-1}$ modulo $\mathcal{I}$.

Proof. The equation (2.3) reduces to (2.9) by applying (2.4). Note that under the isomorphism of the graded algebras $H^{*}\left(Y_{n} ; \mathbb{Z}_{2}\right)$ and $\mathcal{R} / \mathcal{I}, w_{k}\left(Y_{n}\right) \in H^{k}\left(Y_{n} ; \mathbb{Z}_{2}\right)$ corresponds to a polynomial of degree $k$ in $x_{i}, 1 \leq i \leq 2 n$ modulo $\mathcal{I}$ for $1 \leq k \leq n$. Thus we get (2.10) by comparing the degree $k$-terms on either side of (2.9) and (i) follows.

Observe that by applying (2.5), in $\mathcal{R}^{\prime} / \mathcal{I}^{\prime}$ and hence in $\mathcal{R} / \mathcal{I}$, we can substitute for $x_{i}$ in terms of $x_{n+1}, \ldots, x_{n+i}$ modulo $\mathcal{I}$ using the equality

$$
x_{i}=x_{n+i}+\sum_{j=1}^{i-1} c_{j, i} \cdot x_{n+j} .
$$


In particular, $w_{k}\left(Y_{n-1}\right)$ (resp. $\left.w_{k-1}\left(Y_{n-1}\right)\right)$ can be written as a polynomial of degree $k$ (resp. $k-1$ ) in $x_{n+i}, \quad 1 \leq i \leq n-1$. Furthermore, multiplying either side of (2.11) with $x_{n+i}$, along with the equality $x_{i} \cdot x_{n+i}=0$ gives

$$
x_{n+i}^{2}=\sum_{j=1}^{i-1} c_{j, i} \cdot x_{n+i} \cdot x_{n+j}
$$

for $1 \leq i \leq n-1$. It follows that $w_{k}\left(Y_{n-1}\right)$ (resp. $w_{k-1}\left(Y_{n-1}\right)$ ) can be expressed as $\mathbb{Z}_{2}$-linear combinations of square free monomials of degree $k$ (resp. $k-1$ ) in $x_{n+i}, \quad 1 \leq i \leq n-1$ in the algebra $\mathcal{R} / \mathcal{I}$. Now, assertion (ii) follows readily by applying (2.12) again in (2.10).

Corollary 2.4. The $k^{\text {th }}$ Stiefel-Whitney class of $Y_{n}$ can be written in terms of the $(k-1)^{\text {th }}$ Stiefel-Whitney classes of $Y_{n}, \cdots, Y_{1}$ as follows:

$$
w_{k}\left(Y_{n}\right)=\sum_{t=1}^{n-1} w_{k-1}\left(Y_{t}\right) A_{t+1}
$$

where $A_{t}=\sum_{i=1}^{t-1} c_{i, t} x_{n+i}$.

Proof. The proof follows from (2.10) by induction on $n$.

The next proposition has been proved in [12, Lemma 2.2] for real Bott manifolds. We state and prove it here for completeness. Also see 20] for orientability criterion for any small cover.

Proposition 2.5. The real Bott manifold $Y_{n}$ is orientable if and only if the sum of entries in each row of the Bott matrix $C=\left(c_{i, j}\right)$ are zero in $\mathbb{Z}_{2}$, that is,

$$
\sum_{j=1}^{n} c_{i, j} \equiv 0 \bmod 2 \text { for every } 1 \leq i \leq n .
$$

Proof. By putting $k=1$ in (2.10) and by induction on $n$ we get,

$$
w_{1}\left(Y_{n}\right)=\sum_{i=1}^{n-1}\left(\sum_{j=i+1}^{n} c_{i, j}\right) \cdot x_{n+i}=\sum_{i=1}^{n-1}\left(\sum_{j=1}^{n} c_{i, j}\right) \cdot x_{n+i}
$$

where the second equality follows from the fact that $c_{i, j}=0$ for $i \geq j$.

The proposition then follows from the fact that a compact connected differentiable manifold $M$, is orientable if and only if $w_{1}(M)=0$ and that as a $\mathbb{Z}_{2}$-vector space, $H^{1}\left(Y_{n} ; \mathbb{Z}_{2}\right)$ is isomorphic to the subspace of $\mathcal{R} / \mathcal{I}$ freely generated by $x_{n+i}, 1 \leq i \leq n$.

\section{Spin structure on ReAl Bott manifolds}

In this section we give a necessary and sufficient condition in terms of the Bott numbers for a Bott manifold to admit a spin structure (Theorem 3.2).

Definition 3.1. The spinor group $\operatorname{Spin}(n)$ (for $n \geq 3$ ) is the connected double cover of the special orthogonal group $S O(n)$. There exists a short exact sequence of Lie groups

$$
1 \rightarrow \mathbb{Z}_{2} \rightarrow \operatorname{Spin}(n) \stackrel{\lambda}{\rightarrow} S O(n) \rightarrow 1
$$


An oriented Riemannian manifold $X$ is said to admit a spin structure if the oriented frame bundle $F$ associated to its tangent bundle, which is a principal $S O(n)$-bundle, lifts to a principal $\operatorname{Spin}(n)$-bundle. More precisely, if there is a principal $\operatorname{Spin}(n)$-bundle $P$ on $X$ that is a double cover of $F$.

It is further known that an $S O(n)$-bundle admits a spin structure if and only if its second Stiefel-Whitney class is zero ([14, Theorem 1.7, pg 86]). Using this criterion, we give a necessary and sufficient condition, in terms of algebraic identities in the Bott numbers, for an $n$-dimensional orientable Bott manifold to admit a spin structure.

Theorem 3.2. The Bott manifold $Y_{n}$ admits a spin structure if and only if the following two conditions are satisfied:

(1) The row sums of the Bott matrix $C=\left(c_{i, j}\right)$ are even. That is, for every $1 \leq i \leq n$,

$$
\sum_{j=1}^{n} c_{i, j} \equiv 0 \bmod 2
$$

(2) For every $1 \leq j<k \leq n$,

$$
\underbrace{\sum_{r=1}^{n} c_{j, r} c_{k, r}}_{P_{j k}}+\underbrace{c_{j, k} \cdot \sum_{\substack{r, s=1 \\ r<s}}^{n} c_{k, r} c_{k, s}}_{Q_{j k}} \equiv 0 \bmod 2
$$

Proof. Condition (1), which is precisely (2.14), says that $Y_{n}$ is orientable. We know that an orientable manifold admits a spin structure if and only if its second Stiefel-Whitney class vanishes ([14, Theorem 1.7, pg 86]). We will now prove that this is equivalent to condition (2).

By Proposition 2.1 and by equation (2.2),$w\left(Y_{n}\right)$ can be identified with the class in $\mathcal{R} / \mathcal{I}$ of the following term

$$
\prod_{j=1}^{n}\left(1+x_{j}+x_{n+j}+x_{j} \cdot x_{n+j}\right) .
$$

Further, using the relations (2.1) in $\mathcal{I}$ we can rewrite (3.3) as

$$
\prod_{j=2}^{n}\left(1+\sum_{i=1}^{j-1} c_{i, j} \cdot x_{n+i}\right)
$$

Furthermore, Proposition 2.1 gives an isomorphism of graded $\mathbb{Z}_{2}$-algebras, the degree 2 term of $w\left(Y_{n}\right)$, namely $w_{2}\left(Y_{n}\right)$ can be identified with the degree 2 term of expression (3.4) which is the class of the following term in $\mathcal{R} / \mathcal{I}$ :

$$
\sum_{1 \leq j<k \leq n-1}\left(\sum_{r=j+1}^{n} \sum_{\substack{s=k+1 \\ s \neq r}}^{n} c_{j, r} c_{k, s}\right) x_{n+j} x_{n+k}+\sum_{k=1}^{n-2}\left(\sum_{\substack{r, s=k+1 \\ r<s}}^{n} c_{k, r} c_{k, s}\right) x_{n+k}^{2} .
$$

Further, from the identity (3.1) we have,

$$
c_{n-1, n}=0 .
$$


Now, by substituting (2.12) and (3.6) in (3.5) , it follows that $w_{2}\left(Y_{n}\right)$ can be identified with the class of the following term in $\mathcal{R} / \mathcal{I}$ :

$$
\sum_{1 \leq j<k \leq n-2}(\underbrace{\sum_{r=j+1}^{n} \sum_{\substack{s=k+1 \\ s \neq r}}^{n} c_{j, r} c_{k, s}}_{I}+\underbrace{c_{j, k} \cdot \sum_{\substack{r, s=k+1 \\ r<s}}^{n} c_{k, r} c_{k, s}}_{I I}) x_{n+j} x_{n+k} .
$$

The expression $I$ in (3.7) can be rewritten as follows :

$$
\begin{aligned}
\sum_{r=j+1}^{n} \sum_{\substack{s=k+1 \\
s \neq r}}^{n} c_{j, r} c_{k, s} & =\sum_{r=j+1}^{n} c_{j, r}\left(\sum_{s=k+1}^{n} c_{k, s}-c_{k, r}\right) \\
& =\sum_{r=j+1}^{n} c_{j, r} \cdot \sum_{s=k+1}^{n} c_{k, s}-\sum_{r=j+1}^{n} c_{j, r} c_{k, r} \\
& =\sum_{r=1}^{n} c_{j, r} c_{k, r}=: P_{j k}
\end{aligned}
$$

The last equality in (3.8) follows by condition (1) and the fact that $c_{i, j}=0$ for $i \geq j$.

Again, using $c_{i, j}=0$ for $i \geq j$, the expression $I I$ in (3.7) can be rewritten as,

$$
c_{j, k} \cdot \sum_{\substack{r, s=1 \\ r<s}}^{n} c_{k, r} c_{k, s}=: Q_{j k} .
$$

Further, it follows by the definition of $C$ and by (3.1) that,

$$
P_{j n-1}=P_{j n}=Q_{j n-1}=Q_{j n}=0 .
$$

Thus by (3.8), (3.9) and (3.10) it follows that $w_{2}\left(Y_{n}\right)$ can be identified with the class of the following term in $\mathcal{R} / \mathcal{I}$ :

$$
\sum_{1 \leq j<k \leq n}\left(P_{j k}+Q_{j k}\right) x_{n+j} x_{n+k}
$$

Further, as a graded $\mathbb{Z}_{2}$-vector space $H^{2}\left(Y_{n} ; \mathbb{Z}_{2}\right)$ is isomorphic to the subspace of $\mathcal{R} / \mathcal{I}$ freely generated over $\mathbb{Z}_{2}$ by the classes of $x_{n+j} x_{n+k}, 1 \leq j<k \leq n$. Hence the theorem.

Remark 3.3. The only oriented 2-dimensional real Bott manifold is the torus, which is classically known to be spin. The 3 -dimensional oriented real Bott manifolds $Y_{3}$, correspond to the following two Bott matrices :

$$
\left(\begin{array}{lll}
0 & 0 & 0 \\
0 & 0 & 0 \\
0 & 0 & 0
\end{array}\right) \quad\left(\begin{array}{lll}
0 & 1 & 1 \\
0 & 0 & 0 \\
0 & 0 & 0
\end{array}\right)
$$


Here, we immediately see that $w_{2}\left(Y_{3}\right)=0$ and hence $Y_{3}$ admits a spin structure. This is a special case of the well known more general result of Steenrod that an oriented threefold is parallelizable.

Example 3.4. The 4-dimensional Bott manifolds admitting spin structure correspond to the following list of associated Bott matrices :

$$
\begin{aligned}
& \left(\begin{array}{llll}
0 & 0 & 0 & 0 \\
0 & 0 & 0 & 0 \\
0 & 0 & 0 & 0 \\
0 & 0 & 0 & 0
\end{array}\right) \quad\left(\begin{array}{llll}
0 & 0 & 0 & 0 \\
0 & 0 & 1 & 1 \\
0 & 0 & 0 & 0 \\
0 & 0 & 0 & 0
\end{array}\right) \quad\left(\begin{array}{llll}
0 & 0 & 1 & 1 \\
0 & 0 & 0 & 0 \\
0 & 0 & 0 & 0 \\
0 & 0 & 0 & 0
\end{array}\right) \quad\left(\begin{array}{llll}
0 & 0 & 1 & 1 \\
0 & 0 & 1 & 1 \\
0 & 0 & 0 & 0 \\
0 & 0 & 0 & 0
\end{array}\right) \\
& \left(\begin{array}{llll}
0 & 1 & 1 & 0 \\
0 & 0 & 0 & 0 \\
0 & 0 & 0 & 0 \\
0 & 0 & 0 & 0
\end{array}\right) \quad\left(\begin{array}{llll}
0 & 1 & 1 & 0 \\
0 & 0 & 1 & 1 \\
0 & 0 & 0 & 0 \\
0 & 0 & 0 & 0
\end{array}\right) \quad\left(\begin{array}{llll}
0 & 1 & 0 & 1 \\
0 & 0 & 0 & 0 \\
0 & 0 & 0 & 0 \\
0 & 0 & 0 & 0
\end{array}\right) \quad\left(\begin{array}{llll}
0 & 1 & 0 & 1 \\
0 & 0 & 1 & 1 \\
0 & 0 & 0 & 0 \\
0 & 0 & 0 & 0
\end{array}\right)
\end{aligned}
$$

Remark 3.5. Note that the above list of Bott matrices exhausts all orientable 4-dimensional real Bott manifolds. Thus it follows that every orientable 4-dimensional real Bott manifold is also spin. Moreover, it is known that a 4-manifold $M$, is parallelizable if and only if it admits a spin structure (i.e $w_{1}(M)=w_{2}(M)=0$ ) and has vanishing Euler characteristic and signature $(\chi(M)=\sigma(M)=0)$ (see [10, Section 4] and [13, p. 699]). Moreover, by Hirzebruch signature formula, $\sigma(M)=\frac{1}{3} p_{1}(M)[M]$, where $p_{1}(M)$ is the first Pontrjagin class and $[M]$ the fundamental class of $M$. Now, a real Bott manifold has vanishing Euler characteristic (since $\chi\left(Y_{n}\right)=\chi\left(Y_{n-1}\right) \cdot \chi\left(S^{1}\right)$ and $\chi\left(S^{1}\right)=0$ ) and vanishing Pontrjagin classes by [ 6 , Corollary 6.8 (i)]. Thus it follows that a 4-dimensional real Bott manifold is orientable if and only if it is parallelizable. Further, it corresponds to one of the eight Bott matrices in the above list.

The following example shows that this is not the case in dimensions 5 and higher. Indeed there are $n$-dimensional Bott manifolds which are orientable but not spin when $n \geq 5$.

Example 3.6. Let $Y_{n}$ be the $n$-dimensional Bott manifold, $n \geq 5$, associated to the Bott numbers $c_{1,2}=1, c_{1, n-2}=1 ; c_{n-2, n-1}=1, c_{n-2, n}=1$ and $c_{i, j}=0$ otherwise. These numbers clearly satisfy (3.1) but not (3.2). Indeed in this case, when $j=1$ and $k=n-2$, the left hand side of (3.2) is $c_{1, n-2} c_{n-2, n-1} c_{n-2, n} \equiv 1 \bmod 2$.

Definition 3.7. We call the Bott matrix $C$ spin if and only if the associated Bott manifold $Y_{n}=Y_{n}(C)$ is spin.

Definition 3.8. Let $R_{i}$ denote the $i$ th row vector $\left(0, \ldots, 0,0=c_{i, i}, c_{i, i+1}, c_{i, i+2}, \ldots, c_{i, n}\right)$ of $C$. For every $1 \leq j<k \leq n$, we define another $n \times n$ Bott matrix $C_{j k}$ with $R_{j}$ as the $j$ th row and $R_{k}$ as the $k$ th row and remaining rows with all entries 0 . 
Corollary 3.9. The Bott matrix $C$ is spin if and only if $C_{j k}$ is spin for every $1 \leq j<k \leq n$.

Proof. From Theorem 3.2, a necessary and sufficient condition for $C$ to be spin is that the entries $c_{i, j}, 1 \leq j \leq n$ on the row $R_{i}$ for every $1 \leq i \leq n$ satisfy (3.1) and further, the entries $c_{j, r}$, $1 \leq r \leq n$ of $R_{j}$ and $c_{k, s}, 1 \leq s \leq n$ of $R_{k}$ for every $1 \leq j<k \leq n$ satisfy (3.2).

Again by Theorem 3.2, it follows that the necessary and sufficient condition for the Bott matrix $C_{j k}$ to be spin is that the entries $c_{j, r}, 1 \leq r \leq n$, of the $j$ th row and the entries $c_{k, s}$, $1 \leq s \leq n$, of the $k$ th row of $C_{j k}$, satisfy (3.1) and (3.2). This can be readily seen because any row of $C_{j k}$, other than the $j$ th or $k$ th row, has all entries as 0 . Thus the entries on the $i$ th row of $C_{j k}$ where $i \neq j, k$, trivially satisfy (3.2). Moreover, if either $i \neq j, k$ or $l \neq j, k$ and $1 \leq i<l \leq n$, the entries of $C_{j k}$, on the $i$ th and the $l$ th row trivially satisfy (3.2). Hence the corollary.

We state the following proposition without proof:

Proposition 3.10. The $n$-dimensional Bott manifold can alternately be seen as the total space of a fibre bundle over $S^{1}$ with fibre an $(n-1)$-dimensional Bott manifold corresponding to the Bott matrix $C^{1}$ of size $(n-1) \times(n-1)$, defined by deleting the first row and first column of $C$.

In this convention, we shall denote the $n$-dimensional Bott manifold by $Z_{n}$ and the fibre, which is the $(n-1)$-dimensional real Bott manifold associated to the matrix $C^{1}$, by $Z_{n-1}$. We can iterate this process and view $Z_{n-1}$ again as a fibre bundle over $S^{1}$ with fibre $Z_{n-2}$ which is the $(n-2)$-dimensional real Bott manifold associated to the matrix $C^{2}$ of size $(n-2) \times(n-2)$, obtained by deleting the first and the second rows and columns of $C$. Continuing this process we finally get that $Z_{2}$ is a two dimensional Bott manifold associated to the Bott matrix $C^{n-2}$, obtained by deleting the first $n-2$ rows and columns of $C$. Then $Z_{2}$ is a fibre bundle over $S^{1}$ with fibre $Z_{1} \simeq S^{1}$.

Corollary 3.11. The $n$-dimensional real Bott manifold $Z_{n}$ is orientable (respectively spin) implies that the successive fibres $Z_{n-1}, Z_{n-2}, \cdots, Z_{2}$ in the above iterated construction are all orientable (respectively spin).

Proof. This follows from (3.1) and (3.2) since the Bott matrix corresponding to $Z_{k}$ is $C^{n-k}$ which is the matrix obtained from $C$ by deleting the first $k$ rows and $k$ columns.

3.1. Spin condition for a more generally defined real Bott manifold. We recall here that Choi, Masuda and Oum give a more general definition of a Bott matrix in [2]. They call a square matrix $B$ to be a Bott matrix if there exists a permutation matrix $P$ and a strictly upper triangular binary matrix $C$ such that $B=P C P^{-1}$. They denote by $\mathcal{B}(n)$, the set of all such $n \times n$ matrices. Further, it follows from [2, Section 3] that $B$ and $C$ are Bott equivalent. 
Moreover, in [2, Section 2] they also give a construction of a real Bott manifold $M(B)$ associated to $B$. In particular, when $B$ is a strictly upper triangular binary matrix then $M(B)$ is nothing but the associated real Bott manifold.

In the following theorem we give a necessary and sufficient condition for the Bott manifold $M(B)$ to admit a spin structure where $B$ is any matrix in $\mathcal{B}(n)$.

Theorem 3.12. The real Bott manifold $M(B)$ associated to $B=\left(b_{i, j}\right) \in \mathcal{B}(n)$ admits a spin structure if and only if the entries $b_{i, j}$ satisfy the following identities :

(1) For $1 \leq i \leq n$,

$$
\sum_{j=1}^{n} b_{i, j} \equiv 0 \bmod 2
$$

(2) For $1 \leq j<k \leq n$,

$$
\sum_{r=1}^{n} b_{j, r} b_{k, r}+b_{j, k} \cdot \sum_{\substack{r, s=1 \\ r<s}}^{n} b_{k, r} b_{k, s} \equiv 0 \bmod 2 .
$$

Proof. Let $B \in \mathcal{B}(n)$. Let $P$ be an $n \times n$ permutation matrix such that $B=P C P^{-1}$ for an $n \times n$ strictly upper triangular binary matrix $C$. Let $C=\left(c_{i, j}\right)$ and $\sigma \in S_{n}$ be the permutation corresponding to $P$. Note that by $[2,(3.1)]$,

$$
b_{\sigma(i), \sigma(j)}=c_{i, j} \quad \text { for } \quad 1 \leq i, j \leq n
$$

Now, by [2, Theorem 1.6], since $B$ and $C$ are Bott equivalent, $M(B)$ and $M(C)$ are affinely diffeomorphic. In particular, $M(C)$ is spin if and only if $M(B)$ is spin. The proof of the theorem now follows by substituting (3.14) in (3.1) and (3.2).

We derive the following corollary, analogous to Corollary 3.9, We wish to remark here that this result has been proved in [8, Theorem 1.2] using different techniques. We omit the proof which is similary to that of Corollary [3.9.

Corollary 3.13. The Bott matrix $B$ is spin if and only if $B_{j k}$ is spin for every $1 \leq j<k \leq n$, where $B_{j k}$ is the $n \times n$ matrix having same $j$ th and $k$ th row as $B$ and all other rows zero.

Remark 3.14. Recall that, by [2, Table 1], [12, Section 7], 21, Section 3], any $B \in \mathcal{B}(4)$ with $M(B)$ orientable, is Bott equivalent to one of the following three Bott matrices :

$$
\left(\begin{array}{llll}
0 & 0 & 0 & 0 \\
0 & 0 & 0 & 0 \\
0 & 0 & 0 & 0 \\
0 & 0 & 0 & 0
\end{array}\right) \quad\left(\begin{array}{llll}
0 & 1 & 0 & 1 \\
0 & 0 & 1 & 1 \\
0 & 0 & 0 & 0 \\
0 & 0 & 0 & 0
\end{array}\right) \quad\left(\begin{array}{llll}
0 & 0 & 0 & 0 \\
0 & 0 & 1 & 1 \\
0 & 0 & 0 & 0 \\
0 & 0 & 0 & 0
\end{array}\right)
$$


Also, by [21, Section 3] and [2, Table 1], any $B \in \mathcal{B}(5)$ with $M(B)$ orientable, is Bott equivalent to one of the following eight Bott matrices :

$$
\begin{aligned}
& \left(\begin{array}{lllll}
0 & 0 & 0 & 0 & 0 \\
0 & 0 & 0 & 0 & 0 \\
0 & 0 & 0 & 0 & 0 \\
0 & 0 & 0 & 0 & 0 \\
0 & 0 & 0 & 0 & 0
\end{array}\right) \quad\left(\begin{array}{lllll}
0 & 0 & 0 & 0 & 0 \\
0 & 0 & 1 & 1 & 0 \\
0 & 0 & 0 & 1 & 1 \\
0 & 0 & 0 & 0 & 0 \\
0 & 0 & 0 & 0 & 0
\end{array}\right) \quad\left(\begin{array}{lllll}
0 & 1 & 1 & 1 & 1 \\
0 & 0 & 0 & 0 & 0 \\
0 & 0 & 0 & 0 & 0 \\
0 & 0 & 0 & 0 & 0 \\
0 & 0 & 0 & 0 & 0
\end{array}\right) \quad\left(\begin{array}{lllll}
0 & 0 & 0 & 0 & 0 \\
0 & 0 & 0 & 0 & 0 \\
0 & 0 & 0 & 1 & 1 \\
0 & 0 & 0 & 0 & 0 \\
0 & 0 & 0 & 0 & 0
\end{array}\right) \\
& \left(\begin{array}{lllll}
0 & 1 & 1 & 0 & 0 \\
0 & 0 & 1 & 0 & 1 \\
0 & 0 & 0 & 1 & 1 \\
0 & 0 & 0 & 0 & 0 \\
0 & 0 & 0 & 0 & 0
\end{array}\right) \quad\left(\begin{array}{lllll}
0 & 1 & 1 & 0 & 0 \\
0 & 0 & 0 & 0 & 0 \\
0 & 0 & 0 & 1 & 1 \\
0 & 0 & 0 & 0 & 0 \\
0 & 0 & 0 & 0 & 0
\end{array}\right) \quad\left(\begin{array}{lllll}
0 & 0 & 1 & 0 & 1 \\
0 & 0 & 1 & 1 & 0 \\
0 & 0 & 0 & 1 & 1 \\
0 & 0 & 0 & 0 & 0 \\
0 & 0 & 0 & 0 & 0
\end{array}\right) \quad\left(\begin{array}{lllll}
0 & 0 & 1 & 0 & 1 \\
0 & 0 & 1 & 1 & 0 \\
0 & 0 & 0 & 0 & 0 \\
0 & 0 & 0 & 0 & 0 \\
0 & 0 & 0 & 0 & 0
\end{array}\right)
\end{aligned}
$$

We can readily check that all the three matrices in (3.15) are spin and only the first four matrices in (3.16) are spin.

Following [2, Table 1], we let $\mathcal{S p i n}_{n}$ denote the number of $n$-dimensional real spin Bott manifolds up to diffeomorphism. Then, by [2, Example 3.1], Remark 3.3 and Remark 3.14] above, we have the following table :

\begin{tabular}{c|ccccc}
\hline$n$ & 1 & 2 & 3 & 4 & 5 \\
\hline $\mathcal{O}_{n}$ & 1 & 1 & 2 & 3 & 8 \\
\hline $\mathcal{S p i n}_{n}$ & 1 & 1 & 2 & 3 & 4 \\
\hline
\end{tabular}

\section{Digraph Characterization of Spin structure on Real Bott manifolds}

We begin this section by recalling the definition of an acyclic digraph associated to a real Bott manifold. The dictionary between a real Bott manifold and its associated acyclic digraph, via the Bott matrix, established in the work of Choi, Masuda and Oum [2, Section 4], gives a new way of studying the topology of these manifolds by means of the combinatorics of the associated digraph. The main theorem (Theorem 4.5) in this section gives a combinatorial criterion on the acyclic digraph, which characterizes the existence of spin structure on the associated real Bott manifold.

Definition 4.1. A directed graph (digraph) is a tuple $(V, E)$ consisting of a set $V$, of elements, called vertices and a set $E$, of ordered pairs of distinct vertices, called edges.

Definition 4.2. Let $D=(V, E)$ be a digraph with vertices $V=\left\{u_{1}, \cdots, u_{n}\right\}$ and edges $\left(u_{i}, u_{j}\right)$ indexed by an ordered pair of distinct vertices. In particular, we assume that $D$ has no loops and has at most one directed edge between any pair of vertices. The adjacency matrix $A(D)$ associated to $D$ is therefore an $n \times n$ matrix in $M_{n}\left(\mathbb{Z}_{2}\right)$ with diagonal entries zero. Conversely, 
to any such matrix $A=\left(a_{i, j}\right) \in M_{n}\left(\mathbb{Z}_{2}\right)$ we associate a graph with $n$ vertices and an edge from $u_{i}$ to $u_{j}$ if and only if $a_{i, j}=1,1 \leq i, j \leq n$. In particular, given a Bott matrix $C=\left(c_{i, j}\right)$ (see 1.2) we can associate a digraph, $D_{C}$ to it, having $n$ vertices. Moreover, since the matrix is strictly upper triangular, the digraph $D_{C}$ admits an ordering of vertices $u_{1}, \cdots, u_{n}$ such that $i<j$ whenever there is an edge from $u_{i}$ to $u_{j}$. In particular, $D_{C}$ is an acyclic digraph (see [2, Section 4]).

4.1. Notations : Let $D_{C}$ be an acyclic digraph associated to a Bott matrix $C$. For each vertex $u_{i}$ of $D_{C}$ we denote by,

$$
\begin{gathered}
N_{D_{C}}^{+}\left(u_{i}\right):=\left\{u_{j} \mid c_{i, j}=1\right\} \quad \text { and } \quad N_{i}:=\left|N_{D_{C}}^{+}\left(u_{i}\right)\right| \text { is the out degree of } u_{i} \\
N_{D_{C}}^{-}\left(u_{i}\right):=\left\{u_{j} \mid c_{j, i}=1\right\} \quad \text { and } \quad I_{i}:=\left|N_{D_{C}}^{-}\left(u_{i}\right)\right| \text { is the in degree of } u_{i}
\end{gathered}
$$

(see [2, Section 4])

For each pair of vertices $u_{i}, u_{j}$ we further denote by $M_{i j}:=\left|N_{D_{C}}^{+}\left(u_{i}\right) \cap N_{D_{C}}^{+}\left(u_{j}\right)\right|$. More precisely, $M_{i j}$ is the number of vertices $u_{k}$ which are the out neighbours of both $u_{i}$ and $u_{j}$.

The following two lemmas respectively reinterpret the terms $P_{j k}$ and $Q_{j k}$ on the left hand side of the identity (3.2), in terms of the combinatorial data of the digraph.

\section{Lemma 4.3.}

$$
P_{j k}=M_{j k}
$$

Proof. Note that the product $c_{k, r} c_{j, r} \neq 0$ if and only if $c_{k, r}=1=c_{j, r}$, that is if and only if there is an edge from $u_{j}$ to $u_{r}$ as well as $u_{k}$ to $u_{r}$. Thus the sum $\sum_{r=1}^{n} c_{j, r} c_{k, r}=P_{j k}$ counts the number of vertices $\left\{u_{r}\right\}$ that have edges from $u_{j}$ as well as $u_{k}$ coming into it. This number is precisely $M_{j k}$. Hence the lemma.

\section{Lemma 4.4.}

$$
Q_{j k}=c_{j, k} \cdot\left(\begin{array}{c}
N_{k} \\
2
\end{array}\right)
$$

Proof. Note that the number of unordered pairs of distinct edges coming out of $u_{k}$ in $D_{C}$ is precisely $\left(\begin{array}{c}N_{k} \\ 2\end{array}\right)$. Also, the product $c_{k, r} c_{k, s} \neq 0$ if and only if $c_{k, r}=1=c_{k, s}$. Thus the sum $\sum_{\substack{r, s=1 \\ r<s}}^{n} c_{k, s} c_{k, r}=Q_{j k}$ counts the total number of unordered pairs of distinct edges coming out of $u_{k}$. Hence the lemma.

The next theorem reformulates Theorem 3.2 in terms of the associated digraph $D_{C}$.

Theorem 4.5. The n-dimensional Bott manifold $Y_{n}(C)$ admits a spin structure if and only if for the corresponding digraph $D_{C}$ the following two conditions are true:

(1) $N_{k}$ is even for all $1 \leq k \leq n$.

(2) $M_{j k}$ and $c_{j, k} \cdot\left(\begin{array}{c}N_{k} \\ 2\end{array}\right)$ have the same parity for all $1 \leq j<k \leq n$. 
Proof. Note that, the out degree $N_{i}$ of $u_{i}$ is $\sum_{i=1}^{n} c_{i, j}$. Thus the identity (3.1) in the statement of Theorem 3.2 is equivalent to condition (1) above. Furthermore, it follows by Lemma 4.3 and Lemma 4.4 that the identity (3.2) in the statement of Theorem 3.2 is equivalent to condition (2) above. Hence the theorem.

Remark 4.6. Condition (2) in Theorem 4.5 can be made more explicit as follows :

(1) When $N_{k}=4 m$ then $\left(\begin{array}{c}N_{k} \\ 2\end{array}\right)$ is always even. So condition (2) is equivalent to saying that $M_{j k}$ is even.

(2) When $N_{k}=4 m-2$ the $\left(\begin{array}{c}N_{k} \\ 2\end{array}\right)$ is always odd. So condition (2) is equivalent to saying that $M_{j k}$ is even when there is no edge from $u_{j}$ to $u_{k}$ in $D_{C}$ and $M_{j k}$ is odd when there is an edge from $u_{j}$ to $u_{k}$ in $D_{C}$.

We will now look at some examples to illustrate Theorem 4.5.

\section{Example 4.7.}

(1)

$$
C_{1}=\left(\begin{array}{llllll}
0 & 0 & 0 & 0 & 0 & 0 \\
0 & 0 & 0 & 0 & 1 & 1 \\
0 & 0 & 0 & 0 & 1 & 1 \\
0 & 0 & 0 & 0 & 1 & 1 \\
0 & 0 & 0 & 0 & 0 & 0 \\
0 & 0 & 0 & 0 & 0 & 0
\end{array}\right)
$$

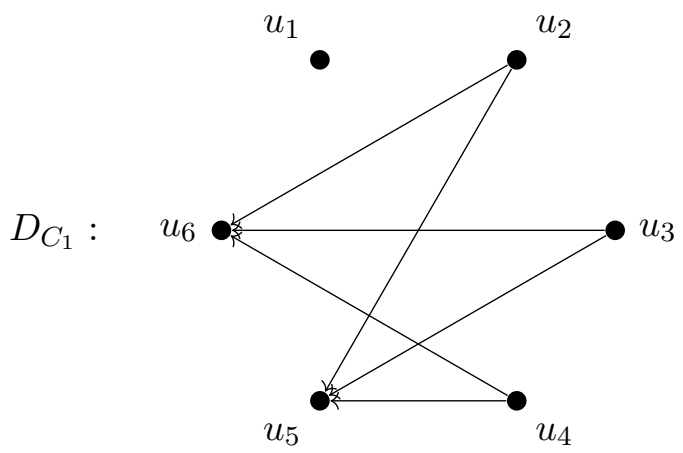

Here we have $N_{D_{C_{1}}}^{+}\left(u_{i}\right)=\left\{u_{5}, u_{6}\right\}$ for $i=2,3,4$ and $N_{D_{C_{1}}}^{+}\left(u_{i}\right)=\emptyset$ for $i=1,5,6$.

Clearly, $N_{i}=\left|N_{D_{C_{1}}}^{+}\left(u_{i}\right)\right|$ is even for all $1 \leq i \leq 5$.

Also, $M_{j k}=\left|N_{D_{C_{1}}}^{+}\left(u_{j}\right) \cap N_{D_{C_{1}}}^{+}\left(u_{k}\right)\right|=2$ for $2 \leq j<k \leq 4$ and $M_{j k}=0$, otherwise.

When $j=2$ and $k=3$ we get that $M_{23}=2$ and $c_{2,3} \cdot\left(\begin{array}{c}N_{3} \\ 2\end{array}\right)=0$ have the same parity.

When $j=2$ and $k=4$ we get that $M_{24}=2$ and $c_{2,4} \cdot\left(\begin{array}{c}N_{4} \\ 2\end{array}\right)=0$ have the same parity.

When $j=3$ and $k=4$ we get that $M_{34}=2$ and $c_{3,4} \cdot\left(\begin{array}{c}N_{4} \\ 2\end{array}\right)=0$ have the same parity.

For other pairs $j<k$ we get $M_{j k}=0$ and $c_{j, k} \cdot\left(\begin{array}{c}N_{k} \\ 2\end{array}\right)=0$ have the same parity.

Thus the associated Bott manifold admits a spin structure.

$$
C_{2}=\left(\begin{array}{llllll}
0 & 1 & 1 & 1 & 1 & 0 \\
0 & 0 & 1 & 0 & 0 & 1 \\
0 & 0 & 0 & 0 & 1 & 1 \\
0 & 0 & 0 & 0 & 0 & 0 \\
0 & 0 & 0 & 0 & 0 & 0 \\
0 & 0 & 0 & 0 & 0 & 0
\end{array}\right)
$$

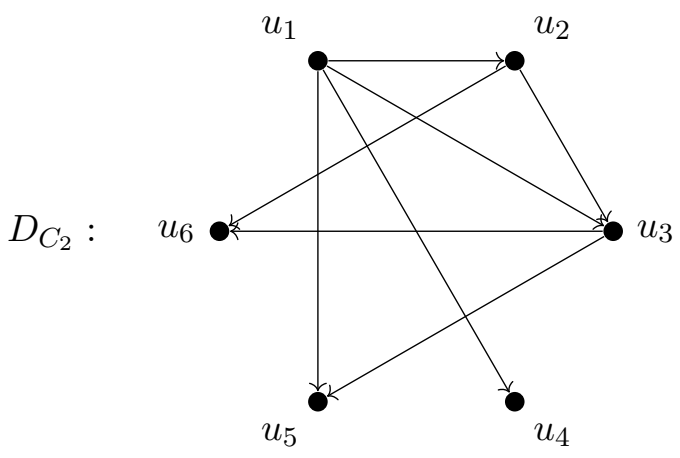


Here we have $N_{D_{C_{2}}}^{+}\left(u_{1}\right)=\left\{u_{2}, u_{3}, u_{4}, u_{5}\right\}, N_{D_{C_{2}}}^{+}\left(u_{2}\right)=\left\{u_{3}, u_{6}\right\}, N_{D_{C_{2}}}^{+}\left(u_{3}\right)=\left\{u_{5}, u_{6}\right\}$ and $N_{D_{C_{2}}}^{+}\left(u_{i}\right)=\emptyset$ for $i=4,5,6$.

Clearly, $N_{i}=\left|N_{D_{C_{2}}}^{+}\left(u_{i}\right)\right|$ is even for all $1 \leq i \leq 6$.

When $j=1$ and $k=2$ we have $M_{12}=\left|N_{D_{C_{2}}}^{+}\left(u_{1}\right) \cap N_{D_{C_{2}}}^{+}\left(u_{2}\right)\right|=\left|\left\{u_{3}\right\}\right|=1$ and $c_{1,2} \cdot\left(\begin{array}{c}N_{2} \\ 2\end{array}\right)=1$ have the same parity.

When $j=1$ and $k=3$ we have $M_{13}=\left|N_{D_{C_{2}}}^{+}\left(u_{1}\right) \cap N_{D_{C_{2}}}^{+}\left(u_{3}\right)\right|=\left|\left\{u_{5}\right\}\right|=1$ and $c_{1,3} \cdot\left(\begin{array}{c}N_{3} \\ 2\end{array}\right)=1$ have the same parity.

When $j=2$ and $k=3$ we have $M_{23}=\left|N_{D_{C_{2}}}^{+}\left(u_{2}\right) \cap N_{D_{C_{2}}}^{+}\left(u_{3}\right)\right|=\left|\left\{u_{6}\right\}\right|=1$ and $c_{2,3} \cdot\left(\begin{array}{c}N_{3} \\ 2\end{array}\right)=1$ have the same parity.

For other pairs $j<k$ we have $M_{j k}=0$ and $c_{j, k} \cdot\left(\begin{array}{c}N_{k} \\ 2\end{array}\right)=0$ have the same parity.

Thus the corresponding Bott manifold admits a spin structure.

$$
C_{3}=\left(\begin{array}{lllll}
0 & 0 & 1 & 0 & 1 \\
0 & 0 & 1 & 1 & 0 \\
0 & 0 & 0 & 1 & 1 \\
0 & 0 & 0 & 0 & 0 \\
0 & 0 & 0 & 0 & 0
\end{array}\right)
$$

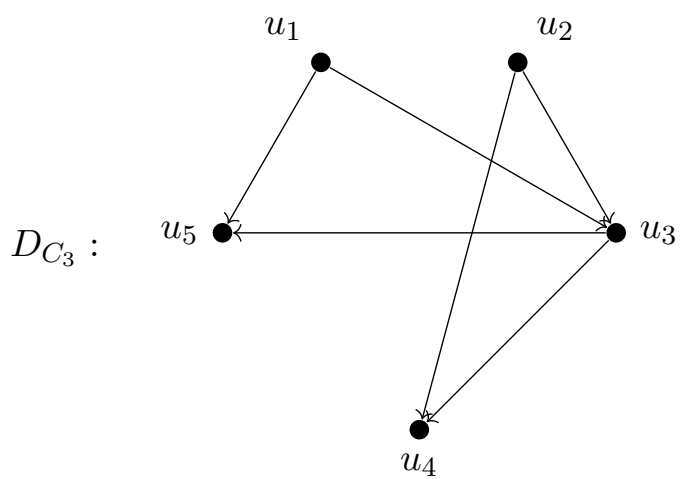

Here we have $N_{D_{C_{3}}}^{+}\left(u_{1}\right)=\left\{u_{3}, u_{5}\right\}, N_{D_{C_{3}}}^{+}\left(u_{2}\right)=\left\{u_{3}, u_{4}\right\}, N_{D_{C_{3}}}^{+}\left(u_{3}\right)=\left\{u_{4}, u_{5}\right\}$ and $N_{D_{C_{3}}}^{+}\left(u_{i}\right)=\emptyset$ for $i=4,5$.

Clearly, $N_{i}=\left|N_{D_{C_{3}}}^{+}\left(u_{i}\right)\right|$ is even for all $1 \leq i \leq 4$.

When $j=1$ and $k=2$ we get $M_{12}=\left|N_{D_{C_{3}}}^{+}\left(u_{1}\right) \cap N_{D_{C_{3}}}^{+}\left(u_{2}\right)\right|=\left\{u_{3}\right\} \mid=1$ and $c_{1,2} \cdot\left(\begin{array}{c}N_{2} \\ 2\end{array}\right)=0$ do not have the same parity.

Thus the associated Bott manifold does not admit a spin structure.

$$
C_{4}=\left(\begin{array}{lllllll}
0 & 0 & 0 & 0 & 0 & 0 & 0 \\
0 & 0 & 1 & 1 & 1 & 1 & 0 \\
0 & 0 & 0 & 1 & 1 & 1 & 1 \\
0 & 0 & 0 & 0 & 0 & 0 & 0 \\
0 & 0 & 0 & 0 & 0 & 1 & 1 \\
0 & 0 & 0 & 0 & 0 & 0 & 0 \\
0 & 0 & 0 & 0 & 0 & 0 & 0
\end{array}\right)
$$

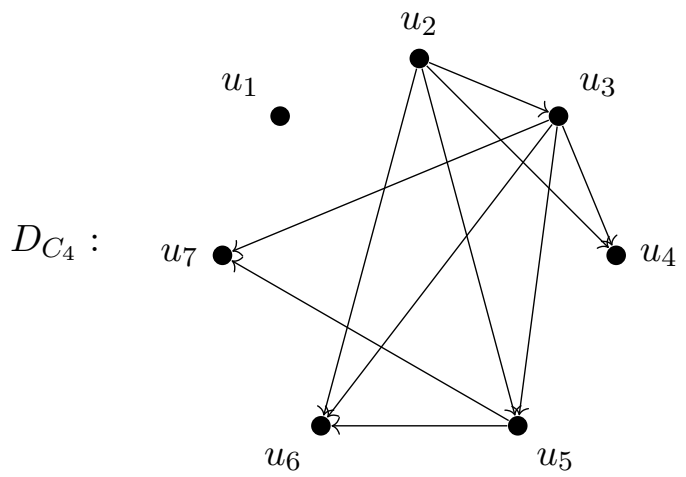

Here we have $N_{D_{C_{4}}}^{+}\left(u_{2}\right)=\left\{u_{3}, u_{4}, u_{5}, u_{6}\right\}, N_{D_{C_{4}}}^{+}\left(u_{3}\right)=\left\{u_{4}, u_{5}, u_{6}, u_{7}\right\}, N_{D_{C_{4}}}^{+}\left(u_{5}\right)=$ $\left\{u_{6}, u_{7}\right\}$ and $N_{D_{C_{4}}}^{+}\left(u_{i}\right)=\emptyset$ for $i=1,4,6,7$

Clearly, $N_{i}=\left|N_{D_{C_{4}}}^{+}\left(u_{i}\right)\right|$ is even for all $1 \leq i \leq 6$ 
When $j=2$ and $k=3$ we get $M_{23}=\left|N_{D_{C_{4}}}^{+}\left(u_{2}\right) \cap N_{D_{C_{4}}}^{+}\left(u_{3}\right)\right|=\left|\left\{u_{3}, u_{5}, u_{6}\right\}\right|=3$ and $c_{2,3} \cdot\left(\begin{array}{c}N_{3} \\ 2\end{array}\right)=6$ do not have the same parity.

Thus the associated Bott manifold does not admit a spin structure.

\section{Higher Stiefel-Whitney Classes and Stiefel-Whitney numbers}

We have the following result for the $(n-1)^{\text {th }}$ Stiefel-Whitney class of $Y_{n}$ :

Theorem 5.1. $\quad$ (i) We have the following formula for $w_{n-1}\left(Y_{n}\right)$ in $H^{*}\left(Y_{n} ; \mathbb{Z}_{2}\right)$ in terms of the Bott numbers $c_{i, j}$ :

$$
w_{n-1}\left(Y_{n}\right)=c_{1,2} \cdot c_{2,3} \cdots c_{n-1, n} \cdot x_{n+1} \cdot x_{n+2} \cdots x_{2 n-1} .
$$

(ii) If $Y_{n}$ is an oriented real Rott manifold then $w_{n-1}\left(Y_{n}\right)=0$.

(iii) We have $w_{n-1}\left(Y_{n}\right)=0$ if and only if there exists a pair of vertices $u_{i}, u_{i+1}$ in the digraph $D_{C}$ with no edge from $u_{i}$ to $u_{i+1}$.

Proof. The proof of (i) follows by induction on $n$ using (2.10) and the fact that in $H^{*}\left(Y_{n} ; \mathbb{Z}_{2}\right)$ the following relations hold :

$$
x_{n+1}^{2}=0 ; x_{n+1} \cdot x_{n+2}^{2}=0 ; x_{n+1} \cdot x_{n+2} \cdot x_{n+3}^{2}=0 ; \cdots ; x_{n+1} \cdot x_{n+2} \cdots x_{2 n-2}^{2}=0 .
$$

From (3.1), $c_{n-1, n}=0$ if $Y_{n}$ is orientable. Hence (ii) follows from (i). Also (iii) follows from (i) and the definition of $D_{C}$.

Remark 5.2. The assertion (ii) of Theorem 5.1 is true for any even dimensional manifold but not in general true when the dimension is odd (see [17, Theorem II and examples on p. 94]).

Remark 5.3. We hope to compute closed formulae for $w_{k}\left(Y_{n}\right)$, for $k \geq 3$ and also characterize their vanishing in terms of the corresponding digraph. For instance when $Y_{n}$ is orientable, by Wu's formula [19, p. 96], $w_{3}\left(Y_{n}\right)=\mathrm{Sq}^{1}\left(w_{2}\left(Y_{n}\right)\right)$. In particular, if $w_{1}\left(Y_{n}\right)=w_{2}\left(Y_{n}\right)=0$ then $w_{3}\left(Y_{3}\right)=0$.

\subsection{Real Bott manifolds bound.}

Definition 5.4. A compact $n$-dimensional manifold $M$ without boundary is null-cobordant if it is diffeomorphic to the boundary of some compact smooth $(n+1)$-dimensional manifold $\mathcal{W}$ with boundary.

Let $w_{k}:=w_{k}\left(Y_{n}\right)$ for $1 \leq k \leq n$. Also let $\mu_{Y_{n}}$ denote the fundamental class of $Y_{n}$ in $H_{n}\left(Y_{n} ; \mathbb{Z}_{2}\right)$. Then

$$
\left\langle w_{1}^{r_{1}} \cdots w_{n}^{r_{n}}, \mu_{Y_{n}}\right\rangle \in \mathbb{Z}_{2}
$$

such that $\sum_{i=1}^{n} i \cdot r_{i}=n$ are the Steifel-Whitney numbers of $Y_{n}$.

Theorem 5.5. Any real Bott manifold is null-cobordant. 
Proof. From the second part of Theorem [2.3, it follows that, any monomial $w_{1}^{r_{1}} \cdots w_{n}^{r_{n}}$ of total dimension $n$ in $\mathcal{R} / \mathcal{I}$ is a $\mathbb{Z}_{2}$-linear combination of square free monomials of degree $n$ in $x_{n+1}, x_{n+2}, \ldots, x_{2 n-1}$. But there are no square free monomials of degree $n$ in $x_{n+j}, 1 \leq j \leq n-1$. Thus the monomial $w_{1}^{r_{1}} \cdots w_{n}^{r_{n}}=0$ in $H^{n}\left(Y_{n} ; \mathbb{Z}_{2}\right)$ so that the associated Stiefel-Whitney number is zero. Therefore by Thom's theorem it follows that $Y_{n}$ is null-cobordant.

Definition 5.6. A compact oriented $n$-dimensional manifold $M^{\prime}$ without boundary is orientedly null-cobordant if it is diffeomorphic to the boundary of some compact smooth $(n+1)$-dimensional oriented manifold $\mathcal{W}^{\prime}$ with boundary.

Let $Y_{n}$ denote an oriented $n$-dimensional real Bott manifold. Let $p_{i}:=p_{i}\left(Y_{n}\right)$ denote the $i$ th Pontrjagin class of $Y_{n}$ in $H^{4 i}\left(Y_{n}, \mathbb{Z}\right)$ and $\mu_{Y_{n}}$ denote the fundamental homology class in $H_{n}\left(Y_{n}, \mathbb{Z}\right)$. Then for each $I=i_{1}, \ldots, i_{r}$ a partition of $k$, the $I$ th Pontrjagin number of $Y_{n}$ is given by

$$
\left\langle p_{i_{1}} \cdots p_{i_{r}}, \mu_{Y_{n}}\right\rangle \in \mathbb{Z}
$$

when $n=4 k$. It is zero when $n$ is not divisible by 4 . Here $v\left[Y_{n}\right]:=\left\langle v, \mu_{Y_{n}}\right\rangle$ denotes the Kronecker index of any $v \in H^{n}\left(Y_{n} ; \mathbb{Z}\right)$ (see [19, p. 185]).

Corollary 5.7. Any oriented real Bott manifold is orientedly null-cobordant.

Proof. Note that [6, Corollary 6.8(i)], implies that all the Pontrjagin numbers of $Y_{n}$ vanish. Moreover, we have shown above in the proof of Theorem 5.5 that all the Stiefel-Whitney numbers of $Y_{n}$ vanish. Thus the corollary follows by Wall's theorem [23, Section 8, Corollary 1].

Remark 5.8. More generally, it follows from [6, Corollary 6.8(i)] and Wall's theorem that if an orientable small cover is null cobordant then it is orientedly null cobordant.

Remark 5.9. There are examples of small covers whose top Stiefel-Whitney class does not vanish. For example the non-orientable surfaces of odd genus ( see [6, Example 1.20]) have non-vanishing second Stiefel-Whitney class.

Remark 5.10. We wish to mention here that Theorem 5.5 was proved jointly with V Uma in [7, Theorem 4.24]. Indeed a more recent preprint has appeared where the same result is proved in [15, Theorem 2.5]. Also using similar techniques Lü extends this result to small covers over $P^{n} \times \Delta^{1}$ where $P^{n}$ is a product of simplices (see [15, Theorem 3.4]). From [15] we came to know that Cheng and Wang [1] and Lü and Tan [16] have proved that any real Bott manifold bounds equivariantly using different methods. However our Corollary 5.7 regarding oriented cobordism is new and could not be explicitly found in the papers [1, 16, 15]

Acknowledgement: I am grateful to my advisor V. Uma for her valuable guidance. I also thank Prof. P. Sankaran for valuable discussions, a careful reading of the manuscript and his 
comments and suggestions and Prof. M. Masuda for his valuable comments and suggestions on earlier versions of the manuscript. I finally thank University Grants Commission (UGC), India for financial assistance.

\section{REFERENCES}

[1] Y. Chen AND Y. WANG, The number of small covers over cubes and the product of at most three simplices up to equivariant cobordism, Proceedings of the Japan Academy, Series A, Mathematical Sciences, 87 (2011), pp. 95-98.

[2] S. Choi, M. Masuda, And S. I. Oum, Classification of real Bott manifolds and acyclic digraphs, Transactions of the American Mathematical Society, 369 (2017), pp. 2987-3011.

[3] S. Choi, M. Masuda, And D. Y. Suh, Topological classification of generalized Bott towers, Transactions of the American Mathematical Society, 362 (2010), pp. 1097-1112.

[4] Y. Civan, Bott towers, crosspolytopes and torus actions, Geometriae Dedicata, 113 (2005), pp. 55-74.

[5] Y. Civan And N. RAY, Homotopy decompositions and K-theory of Bott towers, K-theory, 34 (2005), pp. 1-33.

[6] M. W. Davis and T. Januszkiewicz, Convex polytopes, Coxeter orbifolds and torus actions, Duke Mathematical Journal, 62 (1991), pp. 417-451.

[7] R. Dsouza And V. Uma, Some results on the topology of real Bott towers, arXiv preprint arXiv:1609.05630, (2016).

[8] A. GA̧SIOR, Spin-structures on real bott manifolds, Journal of the Korean Mathematical Society, 54 (2017), pp. 507-516.

[9] M. Grossberg And Y. Karshon, Bott towers, complete integrability, and the extended character of representations, Duke Mathematical Journal, 76 (1994), pp. 23-58.

[10] F. Hirzebruch And H. Hopf, Felder von Flächenelementen in 4-dimensionalen Mannigfaltigkeiten, Mathematische Annalen, 136 (1958), pp. 156-172.

[11] J. JuRkiewicz, Torus embeddings, polyhedra, $k^{*}$-actions and homology, (1985).

[12] Y. Kamishima and M. Masuda, Cohomological rigidity of real Bott manifolds, Algebraic \& Geometric Topology, 9 (2009), pp. 2479-2502.

[13] J. KoRBAš, Distributions, vector distributions, immersions of manifolds in Euclidean spaces, in Handbook of Global Analysis, D. Krupka and D. Saunders, eds., 2008, ch. 13, pp. 665-724.

[14] H. B. Lawson And M. L. Michelsohn, Spin Geometry (PMS-38), vol. 38, Princeton university press, 2016.

[15] Y. L̈̈, On cobordism of generalized (real) Bott manifolds, arXiv preprint arXiv:1710.00562, (2017).

[16] Z. LÜ AND Q. TAN, Small covers and the equivariant bordism classification of 2-torus manifolds, International Mathematics Research Notices, 2014 (2013), pp. 6756-6797.

[17] W. Massey, On the Stiefel-Whitney classes of a manifold, American Journal of Mathematics, 82 (1960), pp. $92-102$.

[18] M. Masuda And T. E. Panov, Semifree circle actions, Bott towers and quasitoric manifolds, Sbornik: Mathematics, 199 (2008), p. 1201.

[19] J. Milnor And J. D. Stasheff, Characteristic Classes.(AM-76), vol. 76, Princeton university press, 2016.

[20] H. Nakayama And Y. Nishimura, The orientability of small covers and coloring simple polytopes, Osaka Journal of Mathematics, 42 (2005), pp. 243-256.

[21] A. NAzRA, Diffeomorphism classes of real Bott manifolds, Tokyo Journal of Mathematics, 34 (2011), pp. 229260.

[22] V. Uma, On the fundamental group of real toric varieties, in Proceedings of the Indian Academy of SciencesMathematical Sciences, vol. 114, Springer, 2004, pp. 15-31. 
[23] C. WALl, Determination of the cobordism ring, Annals of Mathematics, (1960), pp. 292-311.

Department of Mathematics, IIT Madras, Chennai 600036, India

E-mail address: raisadsouza1989@gmail.com 